\title{
Ionic Liquids as Tools in the Production of Smart Polymeric Hydrogels
}

\author{
S. S. SILVA*a,b AND R. L. REIS ${ }^{a, b}$
}

a3B's Research Group - Biomaterials, Biodegradables, Biomimetics, Univ. Minho, Headquarters of the European Institute of Excellence on Tissue Engineering and Regenerative Medicine, AvePark-Parque de Ciencia e

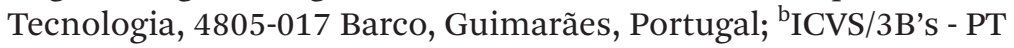
Government Associate Laboratory, Braga/Guimarães, Portugal ${ }^{*}$ E-mail: simonesilva@dep.uminho.pt

\subsection{Introduction}

The application of ionic liquids (ILs) in the development of functional tailor-made materials has grown over the years. This interest has been motivated by the unique physico-chemical properties of ILs, enabling the utilization of IL-based materials in a broad range of fields, namely the chemical, pharmaceutical, energy, and biotechnology fields, among others. ILs are organic salts that contain organic cations such as imidazolium, pyridinium, pyrrolidinium or ammonium derivatives. ${ }^{1,2}$ These can be associated with organic anions such as $\mathrm{CH}_{3} \mathrm{COO}^{-}$or inorganic anions such as $\mathrm{Cl}^{-}, \mathrm{Br}^{-}, \mathrm{I}^{-}$or $\mathrm{BF}^{4-}$ to design a particular IL. ILs are stable in the liquid form at a temperature below $100^{\circ} \mathrm{C}$. They have high ionic conductivity $\left(10^{-4}\right.$ to $\left.10^{-2} \mathrm{~S} \mathrm{~cm}^{-1}\right)$, are inflammable and exhibit negligible vapor pressure. ${ }^{1}$ Furthermore, they also exhibit high thermal (up to $300{ }^{\circ} \mathrm{C}$ )

Smart Materials No. 29

Polymerized Ionic Liquids

Edited by Ali Eftekhari

(C) The Royal Society of Chemistry 2018

Published by the Royal Society of Chemistry, www.rsc.org 
and electrochemical stability $(4-5.7 \mathrm{~V}) .^{3}$ Since ILs have low volatility, they can be recycled through their separation from liquid solutions by evaporation of water or another volatile solvent (e.g. ethanol) and re-used. ${ }^{4}$

The role of ILs not only as a solvent but also as a reaction medium for difficult biomacromolecules has been demonstrated in a number of different publications in the literature. In fact, renewable materials like natural polymers have many properties, such as availability in nature, biocompatibility, and biodegradability, that are useful for the development of renewable smart materials, e.g. hydrogels, membranes, and scaffolds. Despite these benefits, some natural biomacromolecules, such as chitin, chitosan and cellulose, exhibit a lack of solubility in water and organic solvents due to their high crystallinity and rigid inter/intramolecular hydrogen bonds. Therefore, the dissolution, functionalization, and blending of natural biomacromolecules using certain ILs have opened up new possibilities for the potential use of the aforementioned polymers. The dissolution mechanism of these macromolecules with certain ILs has been associated with their ability to disrupt the hydrogen bonds, creating quasi-solid materials termed as ion gels, which offer good mechanical strength and conductivity. Other authors have also reported the application of ILs to extract biopolymers such as agarose ${ }^{5}$ or chitin $^{6}$ from biomass, based on simple, easy and efficient methodologies.

In the literature, it is also possible to find references to the ability of ILs to create hydrogels. ${ }^{7-10}$ Hydrogels are three-dimensional (3D) polymeric networks that are highly swollen and possess hydrophilic character. They are able to absorb large amounts of water or biological fluids. ${ }^{11}$ Polymeric hydrogels have been made using natural (e.g. cellulose, chitin, chitosan, xanthan gum, agarose, or silk fibroin $)^{7,8,10}$ and synthetic polymers, like poly( $N$-isopropyl acrylamide) (PNIPAm) and poly(benzyl methacrylate) (PBzMA), ${ }^{9}$ or a combination of the two. These approaches have also been used to produce stimuli responsive (SR) hydrogels. SR hydrogels can change their physicochemical properties in response to variations in external stimuli, e.g. $\mathrm{pH}$, temperature and magnetic or electric fields. ${ }^{9,11}$

Another possible approach to prepare polymeric hydrogels is the production of poly(ionic liquid)s, PILs, which result from the combination of ILs with polymers. ${ }^{12-14}$ PILs are a subclass of polyelectrolytes that feature IL species in each monomer repeating unit, connected to a polymeric backbone to form a macromolecular architecture. ${ }^{13,15,16}$

In this chapter, we will review the most important and interesting features of polymeric materials, mainly hydrogels resulting from the combination of natural and synthetic polymers prepared in ILs (Figure 11.1), highlighting their potential application in different fields.

\subsection{Polymeric Hydrogels Using Ionic Liquids}

Many reports on ILs and polymers have dealt with the use of ILs as solvents for poorly soluble macromolecules. For instance, different studies have reported that ILs containing strong hydrogen-bond-accepting anions such as 


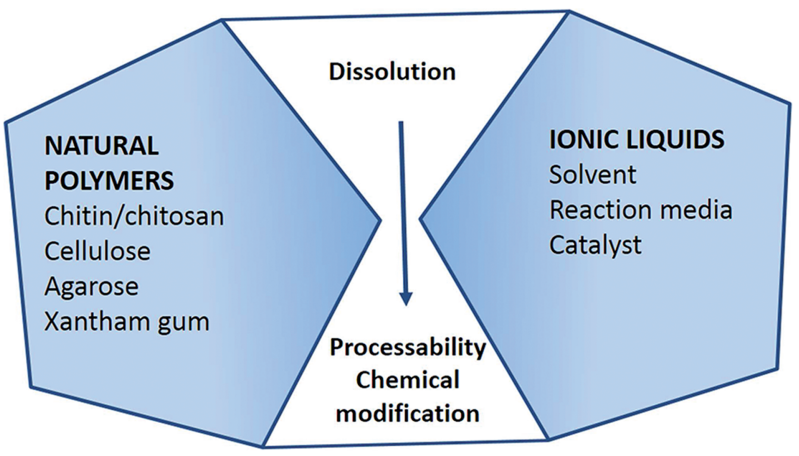

Figure 11.1 Overview of the strategies applied for using natural polymers and ionic liquids for the development of materials.

chloride and acetate anions can dissolve natural polymers. Most of the work in the literature related to biopolymer solubility in ILs is mainly focused on polysaccharides (e.g. agarose, cellulose, chitin/chitosan, xanthan gum and starch) and proteins (gelatin, and silk fibroin). ${ }^{7,17-19}$ Their dissolution is a useful tool as it allows them to be processed into composites, fibres, ion gels, microspheres, and hydrogels.

The preparation of IL-based polymer gels can be based on diverse methodologies, which can be classified into three major types: gelation of ILs within polymers/biopolymers, in situ polymerization of vinyl monomers in ILs, and polymerization of ILs containing polymerizable groups. ${ }^{20}$

In this chapter, the physicochemical properties and characteristics of the natural and synthetic polymers most used in the preparation of polymeric ionogels and hydrogels in ILs are also reviewed.

\subsubsection{Agarose}

Agarose is a linear polysaccharide present in red algae. It is composed of $(1 \rightarrow 3)$ - $\beta$-D-galactopyranose- $(1 \rightarrow 4)$-3,6-anhydro- $\beta$-L-galactopyranose units. $^{21}$ Recent studies have explored the use of ILs as a medium for the efficient extraction of agarose via the dissolution of red algae, under various conditions of heating or microwave irradiation. ${ }^{5}$ This method is easy, simple and highly efficient when compared to the conventional methods of agarose extraction. When solubilized in water, this neutral polysaccharide forms thermoreversible gels, which solidify upon cooling below $35-40{ }^{\circ} \mathrm{C} .{ }^{8}$ To take advantage of its thermoresponsive character, agarose has been associated with chitosan, gelatin, and fibrin. ${ }^{9,22-24}$ These approaches have been utilized in the formation of hydrogels useful for biomedical applications.

The preparation of composites of agarose/chitosan in 1-butyl-3-methylimidazolium chloride (BMIMCl) has been demonstrated. ${ }^{25}$ Besides composite preparation, it is also possible to form ionogels by cooling biopolymer/IL solutions at room temperature (RT). Ionogels can act as smart polymeric 
conducting materials that combine the chemical versatility of an IL with the morphological versatility of a biopolymer. In such ionogels, the IL is immobilized in a way that involves the formation of a three dimensional network. As an example, agarose ionogels have been produced from agarose in ammonium-based ILs and further mixed with imidazolium- or pyridinium-based ILs. ${ }^{26}$ The obtained agarose/IL ionogels present good strength and conductivity when the agarose/IL solutions are cooled to RT. Such ionogels can be suitable for application in electrochemical devices. In another approach, agarose (CAG) ionogels were prepared from a protic-aprotic mixed-IL system (BMIMCl and $N$-(2-hydroxyethyl)ammonium formate). ${ }^{27}$ These ionogels demonstrated superior self-healing properties. In addition, these ionogels were suitable as a flexible solid electrolyte for activated-carbon-based supercapacitors. This type of smart polymeric-material ionogel will also have broad applications in the biomedical and electronic fields.

\subsubsection{Cellulose}

Cellulose is the most abundant polysaccharide in nature. It is constituted by $\beta(1 \rightarrow 4)$-linked $\mathrm{D}$-glucopyranose units. This polymer is recognized for its wide availability, low cost and biocompatibility. Nevertheless, its application has been limited due to its high crystallinity degree and rigid intra/intermolecular hydrogen bonds, resulting in its insolubility in water as well as organic solvents, thus reducing its applications. A series of studies has been initiated on dissolving cellulose in ILs. In 2002, Swatloski and co-workers reported, for the first time, the dissolution of cellulose using an IL, BMIMCl, in relatively high concentrations. ${ }^{28}$ The success of this work stimulated other studies on the dissolution mechanism, processability, and functionalization of cellulose in ILs. Besides BMIMCl, other ILs, namely 1-ethyl-3-methylimidazolium acetate (BMIMAc), 1-allyl-3-methylimidazolium chloride (AMIMCl), and 1-allyl-2,3-dimethylimidazolium bromide (AMIMBr), have been investigated either to dissolve cellulose or as a reaction media for the functionalization of cellulose. ${ }^{28-33}$ Therefore, many aspects related to the dissolution mechanism and interactions of cellulose in ILs have been reviewed by several authors. $^{32,34,35}$ These studies provide molecular insights into the interactions of cellulose with ILs and suggest that hydrogen-bonding is critical to governing/managing cellulose dissolution. In addition, the chemical versatility of both cellulose and ILs has been investigated for the next generation of cellulosic materials for application in different fields. For instance, shapepersistent and tough cellulose hydrogels can be moulded by step-wise solvent exchange from a homogeneous cellulose/IL solution to methanol vapour exposure (Figure 11.2). These tough cellulose hydrogels may find applications as scaffolds for tissue engineering or micro-reactors using microfluidics.

Cellulose and its derivatives are also excellent building blocks for hydrogel fabrication. Kadokawa and co-workers reported a facile approach for the preparation of a flexible cellulose hydrogel material from cellulose/BMIMCl (15 wt\%) solution by keeping it at room temperature for 7 days. ${ }^{29}$ In addition, 

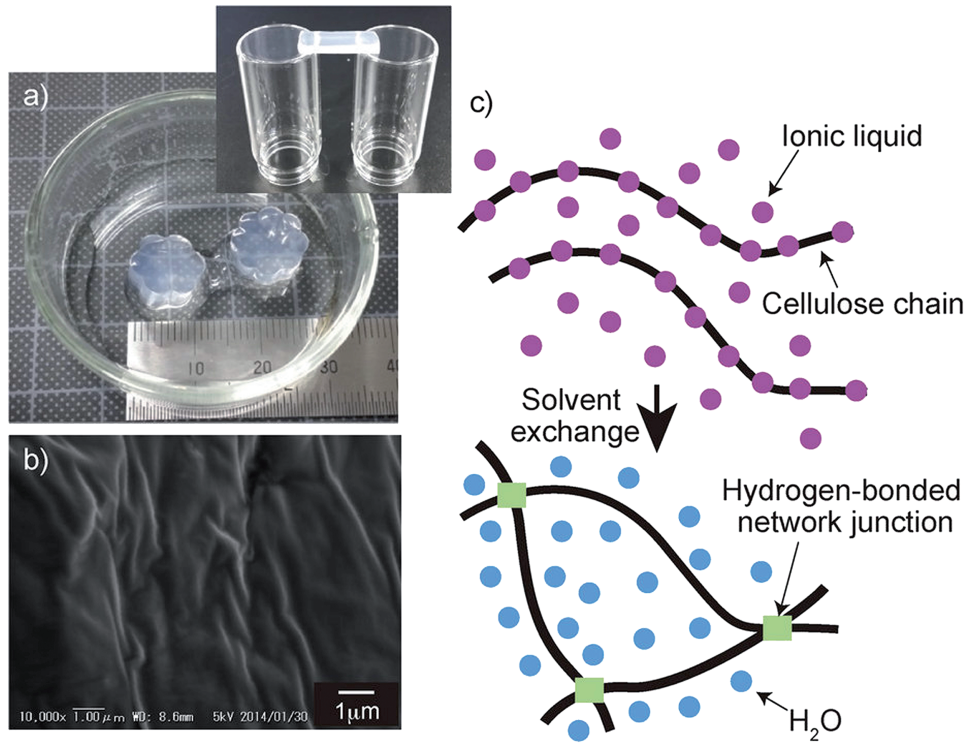

Figure 11.2 (a) Optical images of flower-shaped cellulose hydrogels prepared from a $5 \mathrm{wt} \%$ IL solution of wood pulp. The inset is an optical image of the bridged cellulose hydrogel. (b) SEM image of the surface of the dried cellulose hydrogel. (c) Schematic gelation process of the cellulose solution. Reprinted from Macmillan Publishers Ltd: Scientific Reports, ref. 36. Copyright 2015.

it has been found that the preparation of cellulose/biopolymer composites, e.g. cellulose/collagen hydrogel beads ${ }^{37}$ cellulose/chitosan hydrogel beads and microspheres ${ }^{38}$ and cellulose/agarose beads ${ }^{39}$ relies on the ability of the IL to dissolve cellulose and its regeneration in ethanol or water. These hydrogels have been used for heavy metal adsorption and enzyme immobilization, and as an adsorbent for dye contaminants. Another interesting approach is one described by $\mathrm{Xu}$ and co-workers where cellulose/graphene hydrogels (CGH) were manufactured by regenerating a mixture of wood pulp and reduced graphene oxide (rGO) from a BMIMCl solution using water as a coagulant. ${ }^{40}$ It was found that incorporating rGO into the cellulose matrix improved its mechanical properties, forming highly tough composite hydrogels. These materials hold great potential for applications in wide fields, such as biomedicine and the environment.

\subsubsection{Chitin and Chitosan}

Chitin is the second most abundant polymer after cellulose. ${ }^{21,41}$ This structure is composed of $N$-acetyl-D-glucosamine units linked through $\beta$ - $(1 \rightarrow 4)$-glycosidic linkages. It is found in the shells of crustaceans such as crabs, shrimps, and squid. ${ }^{42}$ Chitin itself has attractive propertiesfor biomedical applications, 
namely the acceleration of woundhealing, tumor cell growth suppression, nontoxicity, and biocompatibility. ${ }^{21,41}$ Despite these properties, and the huge annual production and easy accessibility of biomass resources, chitin has some limitations due to its lack of solubility in water and some organic solvents. The solubility behaviour of chitin is affected by its crystalline structure. $\alpha$-chitin (e.g. chitin isolated from crab and shrimp cells) is formed by strong intermolecular hydrogen bonding, which implies a lack of solubility, while $\beta$-chitin (e.g. chitin extracted from squid pens) has a parallel arrangement, resulting in weaker intermolecular forces, higher reactivity and higher affinity for solvents when compared to $\alpha$-chitin. ${ }^{42}$ Therefore, much attention has been paid to applying ILs as a solvent for chitin as a way to expand its processability and potential uses. In fact, many ILs have been reported in the literature with the ability to dissolve chitin, such as AMIMBr, BMIMAc, 1-ethyl-3-methylimidazolium acetate (EMIMAc), and BMIMCl. ${ }^{43-45}$ It seems that chitin requires a more basic anion such as acetate due to the increased number of hydrogen bond donors and acceptors. ${ }^{46}$ It has also been shown that not only the structure of the anion or the cation is important, but also the features of chitin such as its origin, polymorphic form, molecular weight and degree of acetylation. ${ }^{47}$ The complete dissolution of chitin was achieved using AMIMBr to obtain a flowing solution or an ionic gel depending on the chitin concentration. ${ }^{48}$ It was suggested that the reason behind chitin dissolution in AMIMBr could be related to the IL composition, which is a combination of an allyl substituent and a bromide counter anion on the imidazolium. Despite the promising findings revealed by these studies, it can be difficult to predict the behavior of chitin in a given IL, probably due to the large number of structural variations of ILs.

An IL platform could also lead to economically and environmentally sustainable isolation methodologies and processing of chitin directly from marine sources, e.g. crab shells, replacing the use of hazardous solvents and reducing the energy and time involved in the process. ${ }^{6}$ For instance, Qin et al. have shown that EMIMAc can dissolve raw crustacean shells, leading to a high molecular weight chitin powder, but it can produce fibers directly from the extract solution. ${ }^{49}$ In another study, chitin fibers could be electrospun in a one-pot process directly from a chitin/EMIMAc solution from dried shrimp shells. ${ }^{46}$ Recent studies also demonstrated that ILs, such as hydroxylammonium acetate $\left(\left[\mathrm{NH}_{3} \mathrm{OH}\right][\mathrm{OAc}]\right)$, can reactively demineralize and remove proteins from shrimp shells in an efficient one-pot pulping process, thus allowing the isolation of native chitin with $>80 \%$ purity and a high degree of acetylation and crystallinity. ${ }^{6}$

To date, there are few reports involving the dissolution of chitin to form hydrogels. Shen et al. prepared hydrogels from IL-extracted biopolymers (chitin and cellulose). ${ }^{10}$ These hydrogels exhibited properties substantially different from those made from the commercially available biopolymers. The hydrogels were supercritically dried producing amorphous, lightweight, and porous aerogels. The findings obtained from dye (indigo camine) release suggest the potential use of both chitin and cellulose hydrogels for drug delivery applications. 
Chitosan is a polysaccharide obtained by the alkaline deacetylation of chitin. ${ }^{41}$ This polymer has many properties such as hemostatic action, film-forming ability, chelating activities, adsorption abilities, bacteriostatic action, cationic nature, biodegradability, and biocompatibility. ${ }^{41}$ These particular properties arise from the presence of primary amines along the chitosan backbone. Chitosan is recognized as a key component in materials applied in several fields, namely the food processing, packaging, cosmetic, biomedical, and pharmaceutical fields, and the removal of metal ions from waste water. ${ }^{41,50-54}$

Similar to chitin, research on the use of ILs has also been extended to chitosan. ${ }^{44}$ The processability of chitosan has some limitations due to its insolubility in pure water and common organic solvents. This feature is associated with the strong hydrogen bonding in chitosan and the presence of amorphous and crystalline regions in its structure. A series of ILs, namely 1-EMIMCl, BMIMCl, EMIMAc, and BMIMAc, have been studied for the dissolution and processability of chitosan..$^{44}$ Therefore, the ability of certain ILs, e.g. BMIMCl, to act as a solvent and reaction medium for chitosan occurs through disrupting its hydrogen bonds, allowing its amino group to be exposed completely to react with other chemical reagents easily.

A simple one-step method based on the ability of ILs to co-dissolve cellulose and chitosan has been reported for preparing magnetic biopolymer hybrid hydrogels. ${ }^{55}$ These hydrogels had high adsorption abilities for some heavy metals ions, such as $\mathrm{Cu}^{+2}, \mathrm{Fe}^{+2}$ and $\mathrm{Pb}^{+2}$, and they could be efficiently recycled and reused.

\subsubsection{Silk Fibroin}

Silk fibroin (SF) is a natural protein. It is composed mainly of glycine, alanine, and serine in different percentages. ${ }^{21,56}$ Silks produced by silkworms are biopolymers, and they can be classified into mulberry (Bombyx mori) and non-mulberry (e.g. muga, eri, tasar) silks. ${ }^{57}$

Silk-based materials have been produced into versatile formats such as membranes, nanofibers, scaffolds and hydrogels. ${ }^{58,59}$ The processing of silk proteins into hydrogels, for instance, can be achieved using different methods based on chemical and physical principles. ${ }^{56}$ The methodologies demonstrated that SF hydrogel formation could be dependent on temperature, $\mathrm{pH}$ and protein concentration, among other parameters.

Many reports emphasized the use of certain ILs to solubilize mulberry silkworm Bombyx protein fibroin alone ${ }^{60}$ or in combination with chitosan ${ }^{8}$ and cellulose. ${ }^{61}$ Other studies used ILs to dissolve and process fibroin obtained from the cocoons of mulberry silkworms Antheraea mylitta, ${ }^{7}$ Antheraea assamensis,${ }^{19}$ and Samia/Philosamia ricini (Eri). ${ }^{18}$ However, only a few reports suggested the production of SF hydrogels through the dissolution of SF in certain ILs. SF hydrogel-based constructs were prepared to solubilize both materials in BMIMAc as a common solvent of chitosan/silk (CSF)/BMIMAc blended systems. ${ }^{8}$ The CSF solutions have extended stability and easy 
processability, allowing the moulding of the CSF hydrogels into a range of shapes and sizes. Moreover, in vitro assays showed that these CSF hydrogels provide a good environment for the adhesion and growth of primary human dermal fibroblasts.

Chen and co-workers report the preparation of solutions of regenerated silk fibroin (RSF), obtained from degummed silks of Bombyx mori and Antherae pernyi in EMIMAc. ${ }^{62}$ The results provided an insight into the sol-gel transition of both RSF/EMIMAc/water from the viewpoint of thermodynamics, which is helpful to interpret the conformational changes of RSF, as well as to create a route for the production of biomedical SF hydrogels from $\mathrm{SF} /$ IL solutions.

\subsubsection{Xanthan Gum}

Xanthan gum is an anionic polysaccharide produced by Xanthomonas campestris and a useful food hydrocolloid. It is composed of cellulose-type main chains ( $\beta(1 \rightarrow 4)$-glucan) with trisaccharide side chains (mannose- $\beta(1 \rightarrow 4)$-glucuronic acid- $\beta(1 \rightarrow 2)$-mannose- $\alpha(1 \rightarrow 3)$-) attached to alternate main chain glucose units. ${ }^{63,64}$ Xanthan gum does not readily form hydrogels via the usual gelation processes. In fact, an aqueous dispersion of xanthan gum exhibits only a weak gel-like behaviour in the presence of a sufficient amount of inorganic salt. Since ILs have specific properties to dissolve polysaccharides, they have been considered as an efficient solvent to produce xanthan gum hydrogels. ${ }^{65,66}$ Xanthan gum/BMIMCl gels were prepared by a heating-cooling process. ${ }^{65}$ These gels showed good mechanical properties and a thermally induced shape-memory effect. Later, the gels were converted into xanthan gum hydrogels by soaking them in water, and the resulting hydrogels showed good elasticity (Figure 11.3). Further soaking the obtained hydrogels in $\mathrm{CaCl}_{2}$ aqueous solution gave rise to an ionically cross-linked hydrogel with $\mathrm{Ca}^{+2}$, which exhibited much better mechanical properties and salt concentration-induced swelling/shrinking behaviour. The authors supposed that the shape memory behaviour of the ion gel was created by the effect of ionexchange of the carboxylate metal salts in xanthan gum with BMIMCl during the gel formation.

Setoyama and co-workers prepared cellulose/xanthan gum composite films and hydrogels through gelation with BMIMCl. ${ }^{67}$ In this work, the solutions of cellulose and xanthan gum in BMIMCl were heated and left to gelate at room temperature, followed by Soxhlet extraction with ethanol. Films and hydrogel materials were obtained by the methods of regeneration and exchange of the dispersing media from the ion gels, respectively.

\subsection{Smart Polymeric Hydrogels}

Recent advances in the creation of smart materials involve ILs and PILs that exhibit stimuli responsiveness (e.g. critical solution temperature, $\mathrm{pH}$, magnetic field and thermoreversibility). When such ILs are used to compose new 


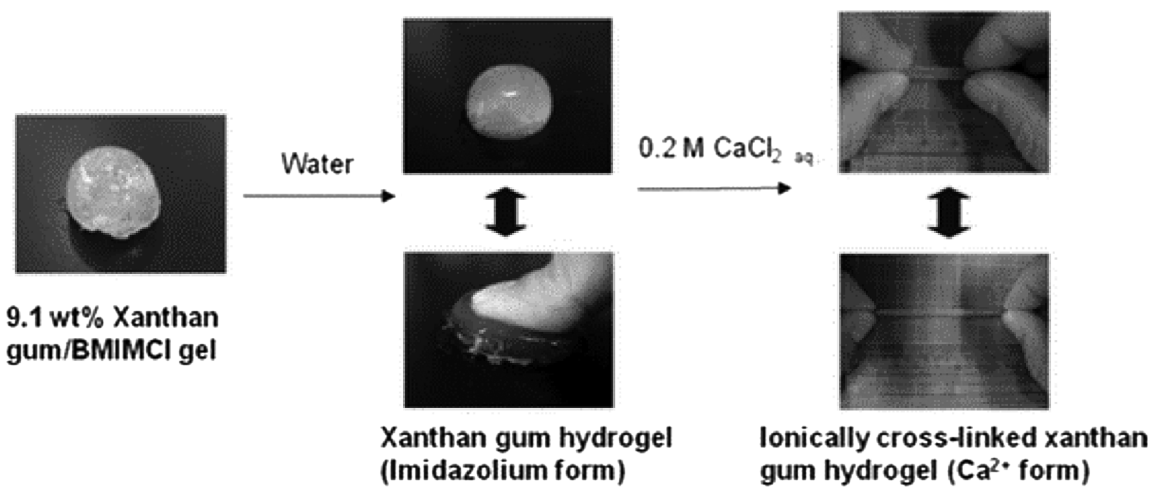

Figure 11.3 Conversion of a $9.1 \mathrm{wt} \%$ xanthan gum/BMIMCl gel into a xanthan gum hydrogel (imidazolium form), which was further converted into an ionically cross-linked hydrogel $\left(\mathrm{Ca}^{+2}\right.$ form $)$ by treatment with a $0.2 \mathrm{M} \mathrm{CaCl}_{2}$ aqueous solution. Reproduced from ref. 65 with permission from The Royal Society of Chemistry.

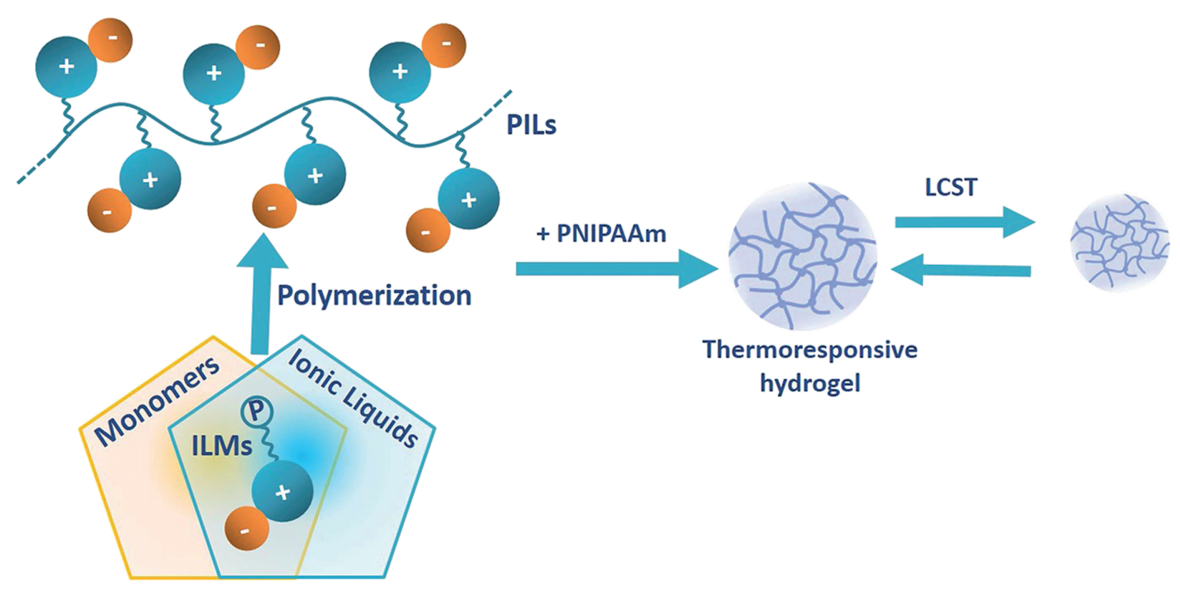

Figure 11.4 Schematic illustration of the relationship between ionic liquids and poly(ionic liquid)s, and the formation of a thermoresponsive hydrogel. ILMs: ionic liquid monomers. "P": polymerizable group. Adapted from Progress in Polymer Science, 38 (7), J. Yuan, D. Mecerreyes, M. Antonietti, Poly(ionic liquid)s: An update, 1009-1036, Copyright (2013) with permission from Elsevier. ${ }^{15}$

polymers, the resulting polymers will often exhibit responsive phenomena. Therefore, SR hydrogels have also been constructed by incorporation of thermosensitive polymers like PNIPAAm and PBzMA into PILs (Figure 11.4). PNIPAAm is the most widely studied thermosensitive polymer in temperature-responsive hydrogels. It shows a lower critical solution temperature (LCST)-type phase separation in aqueous solutions at approximately body temperature. $^{11}$ 
Such materials have potential use in highly diverse areas ranging from controlled release to chemomechanical actuators to supports of catalysis, among other applications.

The combination of ILs with polymers permits the creation of smart soft materials. T. Ueki has reviewed the thermodynamic aspects and recent developments in stimuli-responsive polymers in ILs, as a new class of smart soft materials. ${ }^{68}$ It is suggested that polymers in ILs require prolonged periods to reach thermodynamic equilibrium as a result of the high viscosity of ILs. Therefore, the viscosity of polymer/IL composites must be supressed to achieve a fast response time for the resulting soft materials.

Sui $e t$ al. produced a new class of cross-linkable redox-responsive poly(ferrocenylsilane)-based PILs (PFS-PILs) via a post-polymerization modification approach. ${ }^{69}$ PFS-PILs were obtained by reacting poly[ferrocenyl(3-iodopropyl)-methylsilane] with 1-vinylimidazole at $60{ }^{\circ} \mathrm{C}$ for $24 \mathrm{~h}$. PFS-PILs selfcross-link at low concentrations into nanogels or form macroscopic hydrogel networks at higher concentrations. TEM analysis revealed nanoparticles that were $20-40 \mathrm{~nm}$ in size, corresponding to the cross-linked PFS-PIL nanogels (Figure 11.5). These nanogels were anion-sensitive and redox-responsive. They also proved to be efficient dispersants in the microemulsion polymerization of methyl methacrylate, producing stable PFS-poly(methyl methacrylate) latex suspensions.

PILs have also been used as a route to obtain dual-responsive hydrogels. ${ }^{9}$ These systems are prepared by incorporating two functional monomers that respond to different stimuli, offering additional functional control. Feng and co-workers reported the preparation of dual-responsive hydrogels, which are highly swellable, consisting of thermo-responsive PNIPAM and redox-responsive poly(ferrocenylsilane) (PFS)-based PILs, which are produced by photo-polymerization. ${ }^{9}$ The swelling ability and
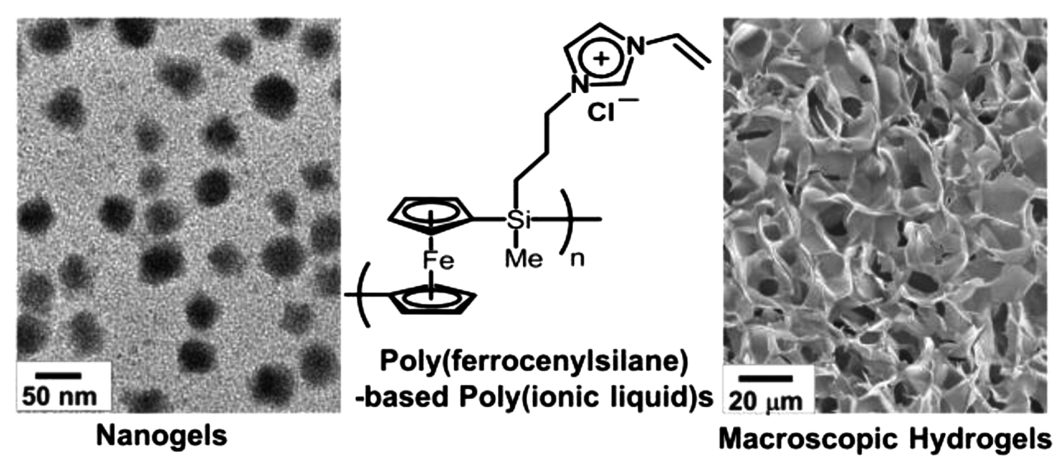

Figure 11.5 Nanogels and macroscopic hydrogels prepared from poly(ferrocenylsilane)-based PILs (chemical structure shown in the middle), which could be cross-linked through the vinylimidazolium functional groups. Reprinted with permission from ref. 69. Copyright (2016) American Chemical Society. 
thermo-responsivity of the hydrogels could betuned by changing the counterion type due to the presence of thepoly(ionic liquid) part. This hydrogel has been employed as a reducing environment for the in situ fabrication of gold nanoparticles (AuNPs), forming AuNP-hydrogel composites. ${ }^{9}$ Another strategy employed for the preparation of responsive hydrogels based on PILs has been described by Tudor et al. ${ }^{13}$ In this work, semi-interpenetrating (sIPN) hydrogels were synthesized by adding increasing amounts of poly $(N$-isopropylacrylamide-co-spiropyran- $c o$-acrylic acid) to a cross-linked PIL matrix. All of the hydrogels, polymerized for approximately $120 \mathrm{~s}$, have different mechanical properties that can be modulated through the content of the copolymer. Gallagher et al. described the sIPN hydrogels prepared using varying concentrations of linear pNIPAAM incorporated into a thermo-responsive PIL hydrogel, namely tributyl-hexyl phosphonium 3 -sulfopropylacrylate (P-SPA). ${ }^{70}$ The swelling and shrinking responses of the obtained hydrogels were modulated by the presence of linear PNIPAAM in the polymer matrix.

Other authors indicated that PNIPAAM-based ionic hydrogels could be synthesized by free-radical polymerization with $N$-isopropylacrylamide as a monomer and imidazolium-based dicationic IL as a cross-linker. ${ }^{12}$ The obtained hydrogels had good swelling properties and exhibited strongly interfacial interaction with anionic dyes such as methyl orange, methyl blue, Congo red, orange G, thymol blue and bromothymol blue in aqueous solution. This behaviour was dependent on the chemical structure of the anionic dyes in solution.

\subsection{Conclusions}

In this chapter, we review the recent advances in the use of ILs in the process of synthesizing polymeric gels (ionogels) and hydrogels. The tunability and versatility of ILs aligned with the features of natural (e.g. chitin or cellulose) and/or synthetic polymers have opened up new routes to process them not only as hydrogels, but also as matrices with different shapes and sizes. In particular, polymeric hydrogels processed in ILs can be useful for a broad range of applications from the removal of anionic dyes to biomaterials. However, concerning biomedical applications, despite the promising findings, little has been reported regarding the in vitro and in vivo biocompatibility of the developed matrices, which could limit their potential use as biomaterials. Most of the research reported suggests the use of ILs as a tool not only in the dissolution/processing of polymers in high value matrices but also to extract biopolymers directly from biomass promoting the economic reduction of the process and an increase in the quality of the obtained biopolymer. The synthesis of PILs has also contributed to certain developments in the creation of responsive hydrogels. Despite this, only a few reports can be found in the literature suggesting that it is at an early stage. However, due to its potential, more developments are expected in the near future. 


\section{List of Abbreviations}

$\begin{array}{ll}\text { AMIMBr } & \text { 1-Allyl-3-methylimidazolium bromide } \\ \text { AMIMCl } & \text { 1-Allyl-3-metylimidazolium chloride } \\ \text { BMIMCl } & \text { 1-Butyl-3-methylimidazolium chloride } \\ \text { BMIMAc } & \text { 1-Ethyl-3-methylimidazolium acetate } \\ \text { CGH } & \text { Cellulose/graphene hydrogels } \\ \text { CSF } & \text { Chitosan/silk } \\ \text { 3D } & \text { Three dimensional } \\ \text { rGO } & \text { Graphene oxide } \\ \text { AuNPs } & \text { Gold nanoparticles } \\ \text { ILS } & \text { Ionic liquids } \\ \text { ILMs } & \text { Ionic liquid monomers } \\ \text { LCST } & \text { Lower critical solution temperature } \\ \text { PILS } & \text { Poly(ionic liquid)s } \\ \text { PFS } & \text { Poly(ferrocenylsilane) } \\ \text { PFS-PILS } & \text { Poly(ferrocenylsilane)-based PILs } \\ \text { PBzMA } & \text { Poly(benzyl methacrylate) } \\ \text { PNIPAm } & \text { Poly(N-isopropyl acrylamide) } \\ \text { P-SPA } & \text { Tributyl-hexyl phosphonium 3-sulfopropylacrylate } \\ \text { RSF } & \text { Regenerated silk fibroin } \\ \text { RT } & \text { Room temperature } \\ \text { SF } & \text { Silk fibroin } \\ \text { SR } & \text { Responsive hydrogels } \\ \text { SIPN } & \text { Semi-interpenetrating } \\ & \end{array}$

\section{Acknowledgements}

The authors are thankful for the financial support from the Portuguese Foundation for Science and Technology (FCT) for the fellowship grant of Simone S. Silva (SFRH/BPD/112140/2015) "Fundo Social Europeu"- FSE and "Programa Diferencial de Potencial Humano POPH".

\section{References}

1. J. Anthony, J. Brennecke, J. Holbrey, E. Maginn, P. Mantz, A. V. Trulove and T. Welton, in Ionic Liquids in Synthesis, ed. P. Wasserscheid and T. Welton, Wiley-VCH, 2002, pp. 41-126.

2. M. Hirao, H. Sugimoto and H. Ohno, J. Electrochem. Soc., 2000, 147, 4168-4172.

3. N. Lourenc,o, A. Nunes, C. Duarte and P. Vidinha, in Applications of Ionic Liquids in Science and Technology, ed. P. S. Handy, 2011, p. 155.

4. P. S. Barber, J. L. Shamshina and R. D. Rogers, Pure Appl. Chem., 2013, 85, 1693-1701.

5. T. Trivedi and A. Kumar, Green Sustainable Chem., 2014, 4, 190-201. 
6. J. L. Shamshina, P. S. Barber, G. Gurau, C. S. Griggs and R. D. Rogers, ACS Sustainable Chem. Eng., 2016, 4, 6072-6081.

7. S. S. Silva, E. G. Popa, M. E. Gomes, M. B. Oliveira, S. Nayak, B. Subia, J. F. Mano, S. C. Kundu and R. L. Reis, Acta Biomater., 2013, 9, 8972-8982.

8. S. S. Silva, T. C. Santos, M. T. Cerqueira, A. P. Marques, L. L. Reys, T. H. Silva, S. G. Caridade, J. F. Mano and R. L. Reis, Green Chem., 2012, 14, 1463-1470.

9. X. L. Feng, K. H. Zhang, P. Chen, X. F. Sui, M. A. Hempenius, B. Liedberg and G. J. Vancso, Macromol. Rapid Commun., 2016, 37, 1939-1944.

10. X. P. Shen, J. L. Shamshina, P. Berton, J. Bandomir, H. Wang, G. Gurau and R. D. Rogers, ACS Sustainable Chem. Eng., 2016, 4, 471-480.

11. J. F. Mano, Adv. Eng. Mater., 2008, 10, 515-527.

12. X. J. Zhou, J. Wang, J. J. Nie and B. Y. Du, Polym. J., 2016, 48, 431-438.

13. A. Tudor, L. Florea, S. Gallagher, J. Burns and D. Diamond, Sensors, 2016, 16, DOI: $10.3390 / \mathrm{s} 16020219$.

14. B. Ziolkowski and D. Diamond, Chem. Commun., 2013, 49, 10308-10310.

15. J. Yuan, D. Mecerreyes and M. Antonietti, Prog. Polym. Sci., 2013, 38, 1009-1036.

16. J. Yuan and M. Antonietti, Polymer, 2011, 52, 1469-1482.

17. R. N. L. de Carvalho, N. M. T. Lourenco, P. M. V. Gomes and L. J. P. da Fonseca, J. Polym. Sci., Polym. Phys., 2013, 51, 817-825.

18. S. S. Silva, N. M. Oliveira, M. B. Oliveira, D. P. S. da Costa, D. Naskar, J. F. Mano, S. C. Kundu and R. L. Reis, Acta Biomater., 2016, 32, 178-189.

19. N. Goujon, R. Rajkhowa, X. Wang and N. Byrne, J. Appl. Polym. Sci., 2013, 128, 4411-4416.

20. J. Lu, F. Yan and J. Texter, Prog. Polym. Sci., 2009, 34, 431-448.

21. S. S. Silva, E. M. Fernandes, S. Pina, J. Silva-Correia, S. Vieira, J. M. Oliveira and R. L. Reis, in Reference Module in Materials Science and Materials Engineering, Elsevier, 2017, pp. 228-252. DOI: 10.1016/B978-012-803581-8.10134-1.

22. S. P. Miguel, M. P. Ribeiro, H. Brancal, P. Coutinho and I. J. Correia, Carbohydr. Polym., 2014, 111, 366-373.

23. R. Imani, S. H. Emami, P. R. Moshtagh, N. Baheiraei and A. M. Sharifi, J. Macromol. Sci., Part B, 2012, 51, 1606-1616.

24. A.-M. Ionescu, M. Alaminos, J. d. 1. C. Cardona, J. d. D. García-López Durán, M. González-Andrades, R. Ghinea, A. Campos, E. Hita and M. d. M. Pérez, J. Mech. Behav. Biomed. Mater., 2011, 4, 1963-1973.

25. T. J. Trivedi, K. S. Rao and A. Kumar, Green Chem., 2014, 16, 320-330.

26. T. J. Trivedi, D. N. Srivastava, R. D. Rogers and A. Kumar, Green Chem., 2012, 14, 2831-2839.

27. T. J. Trivedi, D. Bhattacharjya, J.-S. Yu and A. Kumar, Chemsuschem, 2015, 8, 3294-3303.

28. R. P. Swatloski, S. K. Spear, J. D. Holbrey and R. D. Rogers, J. Am. Chem. Soc., 2002, 124, 4974-4975. 
29. J.-i. Kadokawa, M.-a. Murakami and Y. Kaneko, Carbohydr. Res., 2008, 343, 769-772.

30. X. Y. Hu, K. Hu, L. L. Zeng, M. M. Zhao and H. H. Huang, Carbohydr. Polym., 2010, 82, 62-68.

31. A. Kimura, N. Nagasawa and M. Taguchi, Radiat. Phys. Chem., 2014, 103, 216-221.

32. M. Isik, H. Sardon and D. Mecerreyes, Int. J. Mol. Sci., 2014, 15, 11922.

33. M. Işık, H. Sardon and D. Mecerreyes, in Applications of Ionic Liquids in Polymer Science and Technology, ed. D. Mecerreyes, Springer Berlin Heidelberg, Berlin, Heidelberg, 2015, pp. 135-152.

34. H. Wang, G. Gurau and R. D. Rogers, Chem. Soc. Rev., 2012, 41, 1519-1537.

35. K. M. Gupta and J. Jiang, Chem. Eng. Sci., 2015, 121, 180-189.

36. M. Kimura, Y. Shinohara, J. Takizawa, S. Ren, K. Sagisaka, Y. Lin, Y. Hattori and J. P. Hinestroza, Sci. Rep., 2015, 5, 16266.

37. J. L. Wang, L. G. Wei, Y. C. Ma, K. L. Li, M. H. Li, Y. C. Yu, L. Wang and H. H. Qiu, Carbohydr. Polym., 2013, 98, 736-743.

38. M. F. Li, Z. M. Wang and B. J. Li, Desalin. Water Treat., 2016, 57, 16970-16980.

39. M. H. Kim, S. An, K. Won, H. J. Kim and S. H. Lee, J. Mol. Catal. B: Enzym., $2012,75,68-72$.

40. M. Xu, Q. Huang, X. Wang and R. Sun, Ind. Crops Prod., 2015, 70, 56-63.

41. S. S. Silva, J. F. Mano and R. L. Reis, Crit. Rev. Biotechnol., 2010, 30, 200-221.

42. L. L. Reys, S. S. Silva, J. M. Oliveira, S. G. Caridade, J. F. Mano, T. H. Silva and R. L. Reis, Biomed. Mater., 2013, 8, 045002.

43. J. Kadokawa, Pure Appl. Chem., 2016, 88, 621-629.

44. S. S. Silva, J. F. Mano and R. L. Reis, Green Chem., 2017, 19, 1208-1220.

45. S. S. Silva, A. R. C. Duarte, A. P. Carvalho, J. F. Mano and R. L. Reis, Acta Biomater., 2011, 7, 1166-1172.

46. P. S. Barber, C. S. Griggs, J. R. Bonner and R. D. Rogers, Green Chem., 2013, 15, 601-607.

47. W. T. Wang, J. Zhu, X. L. Wang, Y. Huang and Y. Z. Wang, J. Macromol. Sci., Part B, 2010, 49, 528-541.

48. K. Prasad, M. Murakami, Y. Kaneko, A. Takada, Y. Nakamura and J. Kadokawa, Int. J. Biol. Macromol., 2009, 45, 221-225.

49. Y. Qin, X. M. Lu, N. Sun and R. D. Rogers, Green Chem., 2010, 12, 968-971.

50. I. Younes and M. Rinaudo, Mar. Drugs, 2015, 13, 1133-1174.

51. M. Rinaudo, Prog. Polym. Sci., 2006, 31, 603-632.

52. R. A. A. Muzzarelli, Carbohydr. Polym., 2009, 76, 167-182.

53. M. Cardoso, R. Costa and J. Mano, Mar. Drugs, 2016, 14, 34.

54. M. Prabaharan and J. F. Mano, Macromol. Biosci., 2006, 6, 991-1008.

55. Z. Liu, H. Wang, C. Liu, Y. Jiang, G. Yu, X. Mu and X. Wang, Chem. Commun., 2012, 48, 7350-7352.

56. M. Floren, C. Migliaresi and A. Motta, J. Funct. Biomater., 2016, 7, 26. 
57. S. C. Kundu, B. Kundu, S. Talukdar, S. Bano, S. Nayak, J. Kundu, B. B. Mandal, N. Bhardwaj, M. Botlagunta, B. C. Dash, C. Acharya and A. K. Ghosh, Biopolymers, 2012, 97, 455-467.

58. S. S. Silva, D. Maniglio, A. Motta, J. F. Mano, R. L. Reis and C. Migliaresi, Macromol. Biosci., 2008, 8, 766-774.

59. S. S. Silva, A. Motta, M. T. Rodrigues, A. F. M. Pinheiro, M. E. Gomes, J. F. Mano, R. L. Reis and C. Migliaresi, Biomacromolecules, 2008, 9, 2764-2774.

60. D. M. Phillips, L. F. Drummy, D. G. Conrady, D. M. Fox, R. R. Naik, M. O. Stone, P. C. Trulove, H. C. De Long and R. A. Mantz, J. Am. Chem. Soc., 2004, 126, 14350-14351.

61. Y. B. Yao, E. J. Zhang, X. L. Xia, J. C. Yu, K. J. Wu, Y. M. Zhang and H. P. Wang, Cellulose, 2015, 22, 625-635.

62. C. Zhang, X. Chen and Z. Shao, ACS Biomater. Sci. Eng., 2016, 2, 12-18.

63. K. Limori, K. Yamamoto and J.-I. Kadokawa, Plast. Polym. Technol., 2016, 4, 63-72.

64. L. D. Melton, L. Mindt and D. A. Rees, Carbohydr. Res., 1976, 46, 245-257.

65. H. Izawa, Y. Kaneko and J. Kadokawa, J. Mater. Chem., 2009, 19, 6969-6972.

66. H. Izawa and J.-i. Kadokawa, J. Mater. Chem., 2010, 20, 5235-5241.

67. M. Setoyama, K. Yamamoto and J. Kadokawa, J. Polym. Environ., 2014, 22, 298-303.

68. T. Ueki, Polym. J., 2014, 46, 646-655.

69. X. Sui, M. A. Hempenius and G. J. Vancso, J. Am. Chem. Soc., 2012, 134, 4023-4025.

70. S. Gallagher, L. Florea, K. Fraser and D. Diamond, Int. J. Mol. Sci., 2014, $15,5337$. 